\title{
MOSFIRE: a multi-object near- infrared spectrograph and imager for the Keck Observatory
}

Ian S. McLean, Charles C. Steidel, Keith Matthews, Harland Epps, Sean M. Adkins

Ian S. McLean, Charles C. Steidel, Keith Matthews, Harland Epps, Sean M. Adkins, "MOSFIRE: a multi-object near-infrared spectrograph and imager for the Keck Observatory," Proc. SPIE 7014, Ground-based and Airborne Instrumentation for Astronomy II, $70142 Z$ (23 July 2008); doi: 10.1117/12.788142

Event: SPIE Astronomical Telescopes + Instrumentation, 2008, Marseille, France 


\title{
MOSFIRE: a multi-object near-infrared spectrograph and imager for the Keck Observatory
}

\author{
Ian S. McLean*a ${ }^{\mathrm{a}}$, Charles C. Steidel ${ }^{\mathrm{b}}$, Keith Matthews ${ }^{\mathrm{b}}$, Harland Epps ${ }^{\mathrm{c}}$ and Sean M. Adkins ${ }^{\mathrm{d}}$ \\ ${ }^{a}$ Dept. Physics and Astronomy, Univ of California/Los Angeles, Los Angeles, CA USA 90095-1547 \\ ${ }^{\mathrm{b}}$ Caltech Optical Observatories, California Institute of Technology, Pasadena, CA USA 91125 \\ ${ }^{\circ}$ UCO/Lick Observatory, Univ of California/Santa Cruz, Santa Cruz, CA USA 95064 \\ ${ }^{\mathrm{d}}$ W. M. Keck Observatory, 65-1120 Mamalahoa Highway, Kamuela, HI USA 96743
}

\begin{abstract}
MOSFIRE, the multi-object spectrometer for infra-red exploration, is a near-IR (0.97-2.45 micron) spectrograph and imager for the Cassegrain focus of the Keck I telescope. The optical design provides imaging and multi-object spectroscopy over a field of view (FOV) of 6.14' x 6.14' with a resolving power of R 3,270 for a slit width of 0.7 arc seconds (2.9 pixels along dispersion). The detector is a 2.5 micron cut-off $2 \mathrm{~K}$ x $2 \mathrm{~K} \mathrm{H} 2-\mathrm{RG} \mathrm{HgCdTe}$ array with a SIDECAR ASIC for detector control. A special feature of MOSFIRE is that its multiplex advantage of up to 46 slits is achieved using a cryogenic Configurable Slit Unit (developed in collaboration with the Swiss Centre for Electronics and Micro Technology) reconfigurable under remote control in $<5$ minutes without thermal cycling. Slits are formed by moving opposable bars from both sides of the focal plane. An individual slit has a length of $\sim 7.1$ arc seconds but bar positions can be aligned to make longer slits. A single diffraction grating in two positions along with order-sorting filters gives essentially full coverage of the $\mathrm{K}, \mathrm{H}, \mathrm{J}$ and $\mathrm{Y}$ bands using $3^{\text {rd }}, 4^{\text {th }}, 5^{\text {th }}$ or $6^{\text {th }}$ order respectively. The grating and a mirror are mounted back-to-back, and when the bars are retracted from the FOV MOSFIRE becomes a wide-field imager. A piezo tip-tilt mirror following the field lens is used to provide flexure compensation at the 0.1 pixel level. Two large CCR heads allow the instrument to reach operating temperature in $\sim 7$ days. MOSFIRE is currently in construction.
\end{abstract}

Keywords: Instrumentation, infrared, spectrograph, multi-object

\section{INTRODUCTION}

MOSFIRE is a near-IR $(\sim 0.97-2.45 \mu \mathrm{m})$ multi-object spectrograph and wide-field camera currently under development for the Cassegrain focus of Keck I as part of the third generation instrument program at the W. M. Keck Observatory $(\mathrm{WMKO})^{1}$. The optical design provides imaging or multi-object spectroscopy over a field of view (FOV) of $6.14^{\prime} \times 6.14^{\prime}$ with a resolving power of $\mathrm{R} \sim 3,270$ for a slit width of 0.7 " (2.9 pixels along dispersion). The detector is a $2 \mathrm{~K} \times 2 \mathrm{~K} H 2$ RG HgCdTe array with a $2.5 \mu \mathrm{m}$ cut-off. A special feature of MOSFIRE is that its multiplex advantage of up to 46 slits is achieved using a cryogenic Configurable Slit Unit or CSU (being developed in collaboration with the Swiss Centre for Electronics and Micro Technology, CSEM) that is reconfigurable under remote control in less than 5 minutes without any thermal cycling of the instrument. Slits are formed by moving opposable bars from both sides of the focal plane. An individual slit has a length of $\sim 7.1$ " but bar positions can be aligned to make longer slits. When the bars are removed to their full extent and the grating is changed to a mirror, MOSFIRE becomes a wide-field imager. Two fixed positions for the grating are provided and order-sorting filters give essentially full coverage of the $\mathrm{K}, \mathrm{H}, \mathrm{J}$ and $\mathrm{Y}$ bands using $3^{\text {rd }}, 4^{\text {th }}$, $5^{\text {th }}$ or $6^{\text {th }}$ order respectively. A folding flat following the field lens is equipped with piezo actuators to provide tip/tilt for flexure compensation at the 0.1 pixel level.

MOSFIRE is being developed by the University of California, Los Angeles (UCLA), the California Institute of Technology (CIT) and the University of California, Santa Cruz, (UCSC). The MOSFIRE Co-Principal Investigators are Ian McLean of UCLA and Charles Steidel (CIT), with other leading roles in optics and instrumentation being played by Harland Epps (UCSC) and Keith Matthews (CIT). The project is managed by WMKO Instrument Program Manager, Sean Adkins. MOSFIRE is funded in part by the Telescope System Instrumentation Program (TSIP), operated by AURA and funded by the National Science Foundation and by a private donation to WMKO by Gordon and Betty Moore.

*mclean@astro.ucla.edu

Ground-based and Airborne Instrumentation for Astronomy II, edited by lan S. McLean, Mark M. Casali, Proc. of SPIE Vol. 7014, 70142Z, (2008) · 0277-786X/08/\$18 · doi: 10.1117/12.788142 


\section{SCIENCE REQUIREMENTS}

Top level science requirements for MOSFIRE were established in large part by an ad hoc group reporting to the WMKO Science Steering Committee that considered the minimum scientific requirements of a cost-capped, near-IR imaging spectrometer for the Observatory. Briefly, the principle requirements were:

- Wavelength range of at least 1 to $2.4 \mu \mathrm{m}$, to complement optical imaging spectrometers from 0.3 to $1 \mu \mathrm{m}$.

- Simultaneous wavelength coverage of at least one atmospheric band at a time.

- Spectral resolution of at least $\mathrm{R}=3000$ achieved with a 0.75 " entrance slit.

- Field of view of $>5^{\prime}$ for imaging, $>5^{\prime}$ by $2^{\prime}$ for spectroscopy

- Multiplex factor $>15$

- Background-limited spectroscopy over the full wavelength range in reasonable exposure times.

- Image quality should not degrade the median near-IR seeing by more than $10 \%$, and spectral resolution should not degrade by more than $15 \%$ at the edges of the usable field.

All of these requirements were met or exceeded by the final design which is summarized in Table 1.

Table 1. Summary of the final design parameters for MOSFIRE.

\begin{tabular}{|c|c|}
\hline Wavelength coverage & $\begin{array}{l}0.975 \text { to } 2.40 \mu \mathrm{m} ; 110.5 \text { line } / \mathrm{mm} \text { grating used in orders } 6,5,4,3(\mathrm{Y}, \mathrm{J}, \mathrm{H}, \mathrm{K}) \text {, with } \\
\text { choice of two grating tilts optimized for } \mathrm{H}, \mathrm{K} \text { or } \mathrm{Y}, \mathrm{J} \text {. }\end{array}$ \\
\hline Spectral Resolution & $\mathrm{R} \theta=2290 \Rightarrow \mathrm{R}=3270 \mathrm{w} / 0.7$ " slit, (2.9 pixels) \\
\hline $\begin{array}{l}\text { Simultaneous Wavelength } \\
\text { Range }\end{array}$ & $\begin{array}{l}\text { Coverage is } 0.45 \mu \mathrm{m}(21 \%) \text { in } \mathrm{K} \text { band; } 1.97 \text { to } 2.42 \mu \mathrm{m} \text { for a slit at the center of } \\
\text { the field; } \mathrm{H} \text { band coverage } 1.48 \text { to } 1.81 \mu \mathrm{m} \text { for the same slit }\end{array}$ \\
\hline Pixel Scale & $0.18 "$ in imaging mode \\
\hline Field Size & $\begin{array}{l}\text { 6.12' slit length, } 6.14 \text { ' field for imaging; slits can be placed anywhere within the } \\
\text { imaging field of view. }\end{array}$ \\
\hline Multiplex & $\begin{array}{l}\text { Cryogenic Configurable Slit Unit (CSU): } 46 \text { remotely configurable slits each } \\
7.1 \text { " long; configurable as a smaller number of longer slits. Each slit width can } \\
\text { be adjusted arbitrarily. }\end{array}$ \\
\hline Image Quality & $\begin{array}{l}\text { Design delivers }<0.25 " \text { rms diameter images over } 0.97 \text { to } 2.40 \mu \mathrm{m} \text { with no re- } \\
\text { focus. }\end{array}$ \\
\hline Stability & $\begin{array}{l}<0.3 \text { pixel residual image motion at detector over } 2 \mathrm{hr} \text { observation. Open-loop } \\
\text { flexure compensation using tip/tilt mirror, look-up table. }\end{array}$ \\
\hline Guiding & $\begin{array}{l}\text { Optical CCD guider, offset by } 6.6^{\prime} \text { from the center of the MOSFIRE field, field } \\
\text { of view of } 2.8^{\prime} \text { square on a } 1024 \times 1024 \text { detector with } 0.164 " \text { sampling. }\end{array}$ \\
\hline Throughput: spectroscopy & $>30 \%$ not including telescope, on order blaze \\
\hline Mask configuration time & $<5$ minutes for full re-configuration; goal $<2.5$ minutes \\
\hline Filters & $\begin{array}{l}\text { Minimum complement is order sorting filters for K, H, J, Y; Ks photometric } \\
\text { filter for imaging. Five additional photometric broad or narrow-band filters. }\end{array}$ \\
\hline Lyot stop & $\begin{array}{l}\text { Two position tracking pupil mask [circumscribed or slightly under-sized, } \\
\text { matching hexagonal shape of pupil image (for H, K bands)] }\end{array}$ \\
\hline Detector & $\begin{array}{l}2048 \text { x } 2048 \text { Teledyne } \mathrm{HgCdTe} \mathrm{H} 2-\mathrm{RG} \text { and ASIC; } 18 \mu \mathrm{m} \text { pixels, long- } \\
\text { wavelength cutoff @2.5 } \mu \mathrm{m} \text {; substrate removed; highest QE }\end{array}$ \\
\hline
\end{tabular}




\subsection{Design parameters}

One of the primary design goals was wavelength coverage of a full atmospheric band while preserving the spectral resolution. For reasons of cost, there was a strong impetus to map the spectral format onto a single $2 \mathrm{~K} \times 2 \mathrm{~K}$ detector. A plate scale of $0.18 " /$ pixel was desired to provide reasonable sampling of the typical near-IR seeing of $\sim 0.5$ " for both imaging programs and for spatial resolution along slits. The choice of a reflection grating, with an anamorphic magnification factor of 1.34, provides additional effective focal reduction in the dispersion direction, thus gaining spectral coverage without sacrificing spectral resolution. As a result, one can place slits over a wide range in "x" position within the MOSFIRE field of view and still record most or all of each atmospheric window on a single detector. The effective spatial sampling in the dispersion direction is $0.24 \mathrm{H} /$ pixel, so that the projected slit will be 2.9 and 2.1 pixels respectively for slits of 0.7 " and $0.5 "$. Thus, the adopted configuration meets the desired goals for spectral resolution and spectral coverage on a single detector, eliminating the complication of spectral and/or spatial "gaps" that occur in the case of a detector mosaic and which make data reduction considerably more complex.

\subsection{Performance}

Figure 1 shows the locations of spectra in each of the four MOSFIRE spectral bands for a set of 46 slits randomly placed within a 6.1' $\mathrm{x}$ 3' field. The black square outline represents the $2 \mathrm{~K} \times 2 \mathrm{~K}$ detector. In the $\mathrm{K}$ and $\mathrm{H}$ bands, a grating tilt of 42.614 degrees (the "primary" grating setting) centers the spectra on the detector, and only a small amount of wavelength coverage is lost for slits at the most extreme x-positions. For the $\mathrm{Y}$ and $\mathrm{J}$ bands, the spectra are more optimally placed on the detector using the "secondary" grating tilt of 41.524 degrees - full coverage for each spectrum is obtained for these bands.
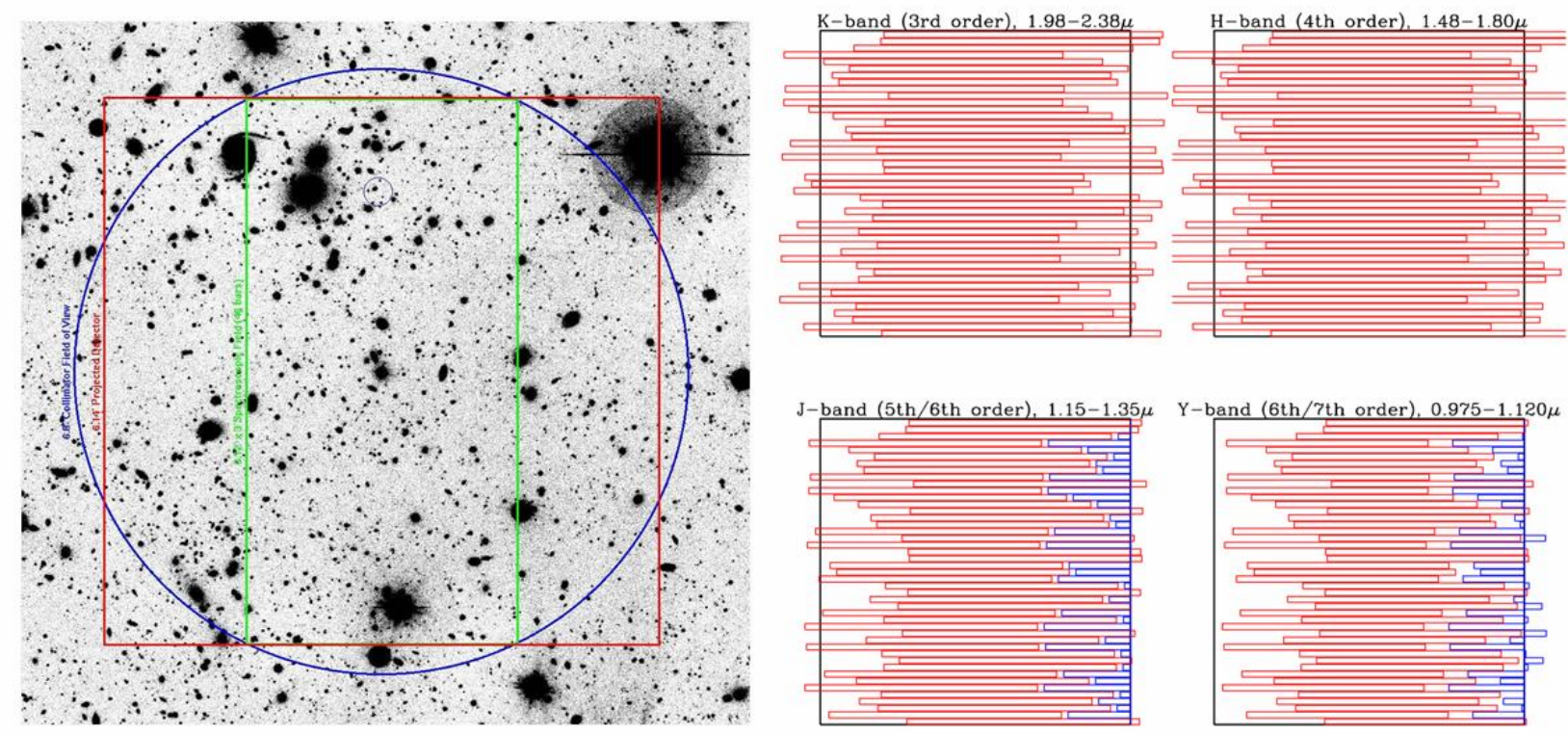

Fig. 1. Left: Imaging field of view (blue circle) and primary spectroscopy field (green rectangle $=6.12^{\prime} \times 3^{\prime}$ ) on projected detector format (red square). Right: 46 randomly generated slits are deployed over the central 6.12' x 3' region. Slit positions are identical for all 4 bands $(\mathrm{K}, \mathrm{H}, \mathrm{J}$ and $\mathrm{Y})$ but the secondary grating tilt was used for $\mathrm{Y}, \mathrm{J}$ spectra.

While the principal reason for adopting a configurable slit unit (CSU) instead of machined or laser-cut focal plane slit masks is to avoid operational issues that would be very costly in the long term, and which would require frequent cryogenic cycling of a separate fore-dewar in order to install and remove focal plane masks, there are also scientific advantages to configuring the masks remotely. For example, it will be possible for users to reconfigure masks in realtime to adjust to changing conditions (e.g. narrowing or widening of slits as seeing conditions change) or to allow for immediate reaction to discoveries or required changes in strategy in the course of executing an observing program. The disadvantages of a CSU, compared to disposable focal plane masks, are that it will not be possible to use "tilted" slits (where the angle of each slit is different to follow the major axis of targets), or to place more than 1 slit on the same row (for higher multiplexing at the expense of spectral coverage). However, the former will not be used much for near-IR work because nodding along the slit is likely to be required for accurate sky subtraction. The CSU has a bar pitch of 5.8 
$\mathrm{mm}$ and this allows an acceptable slit length of $5.1 \mathrm{~mm}$ or $\sim 7.1$ " in the Keck $\mathrm{f} / 15$ focal plane (with about 0.9 " of overlap between bars) and provides for up to 46 slits within the MOSFIRE field of view. For significantly lower target surface density, a smaller number of objects will be accommodated, and adjacent slits can be combined to form a longer slit with no dead regions in between, so that multiple (continuous) slits of arbitrary length can be constructed in $\sim 8$ " intervals. It should be noted that with the same telescope position and PA, only about $10 \%$ fewer objects are observed than in the (more conventional) case where slits could have arbitrary length with a minimum of 7.1"; in other words, the fixed format of the CSU bars makes only a small compromise in terms of the efficiency of observing targets. For targets with lower surface density on the sky, the "quantized" nature of the slit lengths will have an even less important effect on efficiency.

MOSFIRE will be background-limited in all of its modes of operation, and thus the spectral sensitivity is ultimately limited by the intensity of the background and by the precision with which the background can be subtracted. With the exception of the long wavelength end of the K-band window, the background is dominated by atmospheric $\mathrm{OH}$ emission. Unless the spectrograph is very stable over time, spectral regions in the vicinity of bright $\mathrm{OH}$ lines will be rendered unusable by systematic errors in sky subtraction. Our goal for residual flexure after correction is 0.1 pixel $(<3 \%$ of a spectral resolution element) with the maximum acceptable value $<0.3$ pixel $(\sim 10 \%$ of a typical spectral resolution element). This requirement, together with the spectral resolution requirements above, is chosen to maximize the sensitivity and useful spectral regions by getting between $\mathrm{OH}$ lines and then insuring that sky subtraction residuals will be minimized in regions close to $\mathrm{OH}$ lines. The viability of controlling instrument flexure at this level using an open-

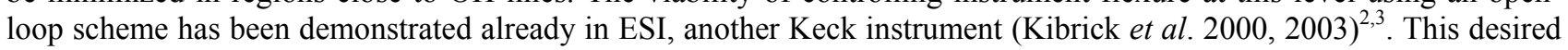
level of stability is also necessary to allow for efficient acquisition of multi-slit or long slit spectroscopic targets, and for the use of calibrations (e.g., flat fields, arc lamps) that may be obtained during the afternoon rather than during the night.

Table 2. MOSFIRE throughput estimates.

\begin{tabular}{|l|l|l|l|}
\hline \multirow{2}{*}{ Component } & \multicolumn{3}{|l|}{ Transmission/Reflectance } \\
\cline { 2 - 4 } & $1.0 \mu \mathrm{m}$ & $1.6 \mu \mathrm{m}$ & $2.2 \mu \mathrm{m}$ \\
\hline Dewar Window (double) & 0.95 & 0.95 & 0.95 \\
\hline Collimator (6 x 2 surfaces) & 0.84 & 0.84 & 0.84 \\
\hline Fold flat (protected Ag) & 0.99 & 0.99 & 0.99 \\
\hline Camera (7 x 2 surfaces) & 0.84 & 0.84 & 0.84 \\
\hline $\begin{array}{l}\text { Internal transmission of all } \\
\text { glasses }\end{array}$ & 0.94 & 0.92 & 0.77 \\
\hline Filter & 0.90 & 0.90 & 0.90 \\
\hline Detector (Hawaii2-RG) & 0.80 & 0.80 & 0.80 \\
\hline Lyot stop (pupil vignetting) & 1.00 & 1.00 & 0.90 \\
\hline $\begin{array}{l}\text { Net Imaging } \\
\text { Throughput }\end{array}$ & $\mathbf{0 . 4 5}$ & $\mathbf{0 . 4 4}$ & $\mathbf{0 . 3 3}$ \\
\hline Grating (on blaze) & 0.65 & 0.75 & 0.83 \\
\hline $\begin{array}{l}\text { Net Spectroscopy } \\
\text { Throughput }\end{array}$ & $\mathbf{0 . 2 9}$ & $\mathbf{0 . 3 3}$ & $\mathbf{0 . 2 7}$ \\
\hline
\end{tabular}

Notes to table: Average reflection loss is $2.4 \%$ per surface for $\mathrm{ZnSe}$ and $1.2 \%$ per surface for all others. The grating efficiency is as built for replicas of the similarly-specified GNIRS grating (order 6, 4, 3 for the 3 entries). As-built results for MOSFIRE components already available suggest that these goals will be achieved or exceeded. 
The detection rate for sky in between $\mathrm{OH}$ lines in the darkest parts of the $\mathrm{Y}, \mathrm{J}$, and $\mathrm{H}$ bands (for even the most optimistic estimates) is $0.3 \mathrm{e}^{-} / \mathrm{s} /$ pixel for a $0.7 "$ slit assuming $\sim 3$ pixel sampling in the dispersion direction $(\mathrm{R}=3,270)$. A dark current of $<0.03 \mathrm{e}-/ \mathrm{s} /$ pixel and an effective read noise below $\sim 5 \mathrm{e}^{-}$would easily result in background-limited performance for most exposures; typical spectroscopic integrations are expected to be in the range $600 \mathrm{~s}$ to $1800 \mathrm{~s}$, but much shorter exposures would be possible without paying a significant detector noise penalty. Table 2 summarizes the estimated throughput of MOSFIRE, showing that with good AR coatings $(<1.5 \%$ average reflection losses per surface $)$ excellent performance is possible. Table 3 gives the estimated sensitivity of MOSFIRE using conservative estimates of the night sky background between $\mathrm{OH}$ emission lines (for spectroscopy) and broadband for imaging.

Table 3. MOSFIRE sensitivity and performance estimates.

\begin{tabular}{|c|c|c|c|c|c|}
\hline Passband & $\begin{array}{l}\text { Spectral Sky } \\
\text { Brightness, } \\
\text { mag arc sec } \\
\\
\text { Vega (AB) }\end{array}$ & $\begin{array}{l}\text { Vega }(A B) \text { mag } \\
\text { for } S / N=10 \text { in } \\
1000 \mathrm{~s}, \mathrm{R}=3270 \\
\text { with } 0.7^{\prime \prime} \text { slit }\end{array}$ & $\begin{array}{l}\text { Line flux for } \\
\mathrm{S} / \mathrm{N}=10 \\
\text { (unresolved line) } \\
\text { in } 1000 \mathrm{~s}, \mathrm{R}=3270 \\
\left(\mathrm{erg} \mathrm{s}^{-1} \mathrm{~cm}^{-2}\right)\end{array}$ & $\begin{array}{l}\text { Imaging Sky } \\
\text { Brightness, } \\
\text { mag arc sec }{ }^{-2} \\
\text { Vega (AB) }\end{array}$ & $\begin{array}{l}\text { Vega }(\mathrm{AB}) \\
\text { mag for } \\
\mathrm{S} / \mathrm{N}=10 \text { in } \\
1000 \mathrm{~s}, \\
\text { broadband }\end{array}$ \\
\hline $\mathrm{Y}(0.97$ to $1.13 \mu \mathrm{m})$ & $17.3(17.9)$ & $20.9(21.5)$ & $0.9 \times 10^{-17}$ & $16.3(16.9)$ & $24.4(25.0)$ \\
\hline $\mathrm{J}(1.15$ to $1.35 \mu \mathrm{m})$ & $16.8(17.7)$ & $20.4(21.3)$ & $0.8 \times 10^{-17}$ & $15.7(16.6)$ & $23.7(24.6)$ \\
\hline $\mathrm{H}(1.48$ to $1.80 \mu \mathrm{m})$ & $16.6(18.0)$ & $20.1(21.5)$ & $0.5 \times 10^{-17}$ & $13.4 \quad(14.7)$ & $22.5(23.9)$ \\
\hline $\mathrm{K}(1.95$ to $2.40 \mu \mathrm{m})$ & $14.4(16.3)$ & $18.6(20.5)$ & $0.9 \times 10^{-17}$ & $13.2(15.0)$ & $21.7(23.6)$ \\
\hline
\end{tabular}

Notes: spectroscopic limits assume $0.05 \mathrm{e}^{-} / \mathrm{s}$ dark current and effective read noise of $4 \mathrm{e}^{-} / \mathrm{pixel}$, with background for spectral regions between $\mathrm{OH}$ lines, evaluated over a 3 pixel resolution element and assuming a $0.5 \operatorname{arcsec}^{2}$ extraction aperture. Imaging limits assume 3 x 3 pixel (0.54") aperture for a point source under good seeing conditions.

Given our choice to use a single reflection grating in multiple orders, order sorting filters are essential to the MOSFIRE design. The minimum filter complement would be passbands designed for spectroscopy in $3^{\text {rd }}, 4^{\text {th }}, 5^{\text {th }}$, and $6^{\text {th }}$ orders of the grating, corresponding closely to the photometric $\mathrm{K}, \mathrm{H}, \mathrm{J}$, and Y passbands. The as-built MOSFIRE filter wheels accommodate a total of 10 filters in two wheels (each carrying 5 filters and an "open"). A $\mathrm{K}_{\mathrm{s}}$ filter for imaging will also be installed. The filters being delivered as part of the MOSFIRE project are summarized in Table 4. Figure 2 shows the as-built combined filter and grating transmissions.

Table 4. MOSFIRE filters and bandpasses.

\begin{tabular}{|l|c|}
\hline Filter & Cut-on/Cut-off $(\boldsymbol{\mu m})$ \\
\hline Yspec & $0.975 / 1.120$ \\
\hline Jspec & $1.150 / 1.350$ \\
\hline Hspec & $1.460 / 1.810$ \\
\hline Kspec & $1.930 / 2.400$ \\
\hline $\mathrm{K}_{\mathrm{s}}$ & $1.990 / 2.310$ \\
\hline
\end{tabular}




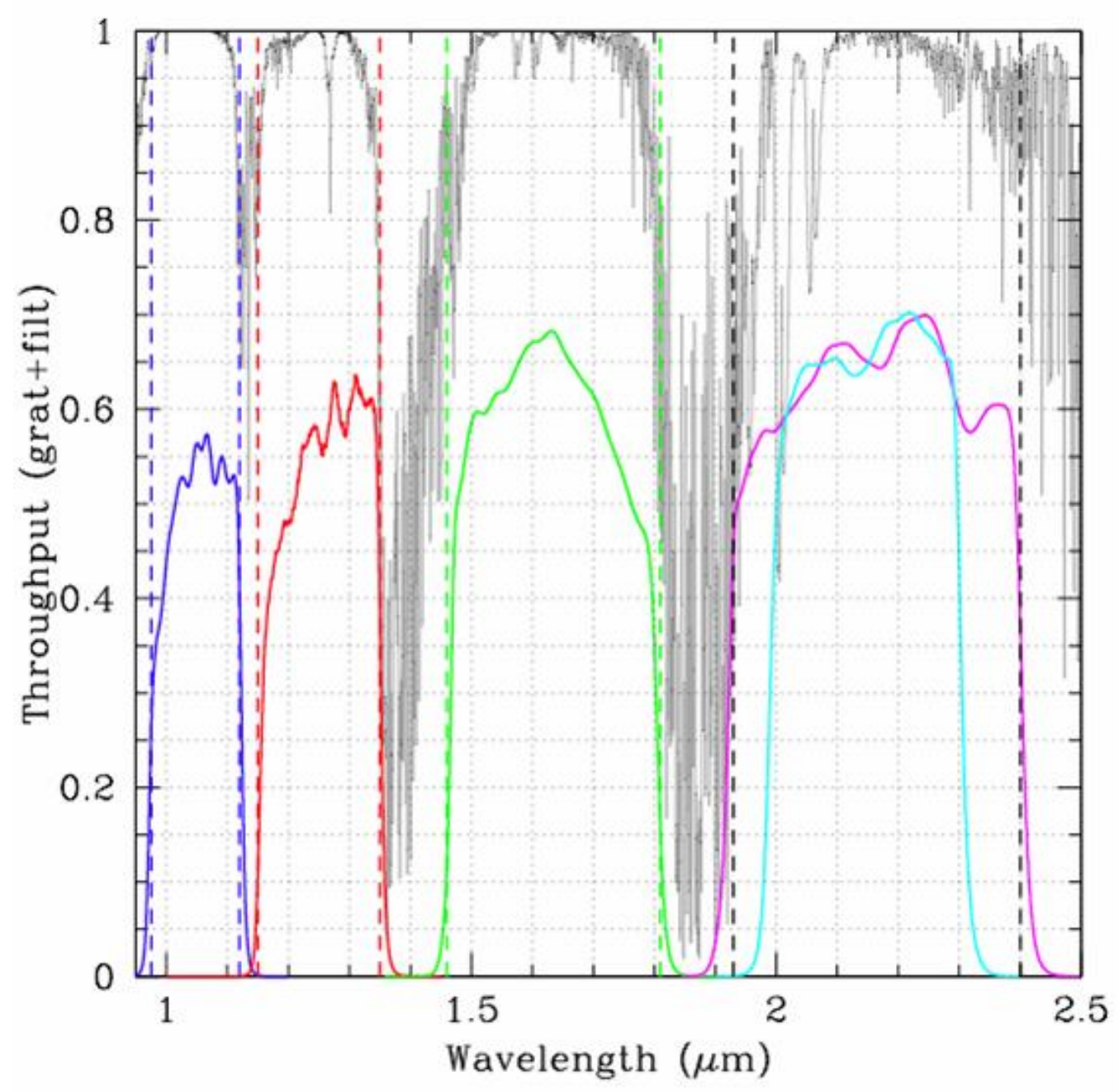

Fig. 2. As-built MOSFIRE grating and spectroscopic (order sorting) filter throughput. The MOSFIRE passbands are shown relative to the near-IR atmospheric transmission on Mauna Kea. A narrower $\mathrm{K}_{\mathrm{s}}$ filter also is included.

The optical design is achromatic but focus adjustment is provided, especially for initial integration and testing. A collimated beam of $125 \mathrm{~mm}$ is required with an accessible pupil for a Lyot stop and mechanism. An external CCD guider (Table 1) with a $1024 \times 1024 \mathrm{CCD}$ having pixels matched to $0.16^{\prime \prime}$ is co-mounted with a calibration lamp unit in front of the MOSFIRE entrance window and protected by a dust cover. To eliminate build up of condensation on the large entrance window a double-window concept is used as described in Sections 2 and 3. The operating temperature of the CSU and optics was chosen to be $120 \mathrm{~K}$, which ensures low thermal emission but optimizes the operation of the closed-cycle refrigerators. Two grating positions give the optimum arrangement for the four spectral regions covered by MOSFIRE, and this feature is achieved without compromising repeatability. A means for compensating for flexure is important and this is solved by splitting the collimator into two sections and using the fold mirror as a tip/tilt mirror.

\section{OPTICAL DESIGN}

The underlying guideline for the MOSFIRE optical design was to keep it mechanically compact and as simple as possible to fabricate. A folded, all-spherical design was adopted for those reasons. Figure 3 shows the Collimator and Camera systems and a depiction of the overall layout. All components in the optical path, including the CSU, are shown. 
A large (388.0 $\mathrm{mm}$ full diameter) entrance window is required to accommodate the $6.8^{\prime}$ diameter field of view. This window is located in a tube or snout at the front of the dewar. In order to withstand the pressure differential under vacuum, while keeping the maximum stress within the "glass" below $\sim 500 \mathrm{psi}$, an Infrasil-302 window about $34.9 \mathrm{~mm}$ thick is used. To prevent condensation from forming as the center of this large window cools by radiation, a second, thinner $(17.0 \mathrm{~mm})$ Infrasil-302 window is located $228.6 \mathrm{~mm}$ away in the vacuum. An aluminum tube and baffles between the windows are resistively heated as described in the mechanical section to provide radiation to maintain the outer window above the average Observatory dome temperature.

The telescope's effective focal length is $149.572 \mathrm{~m}$ as it is used with MOSFIRE, such that the effective f/ratio is $\mathrm{f} / 14.50$. Thus the collimator's focal length must be $1813.0 \mathrm{~mm}$ so as to produce the stipulated $125.0-\mathrm{mm}$ beam diameter. The collimator is a critical optical component. It must capture a physically large, $6.8^{\prime}$ diameter field of view and it must also produce a sharply focused, accessible pupil image so that a cold Lyot stop (mask) can be inserted to eliminate stray thermal radiation. We have optimized the design to the adopted operating temperature of $\mathrm{T}=120.0 \mathrm{~K}$.
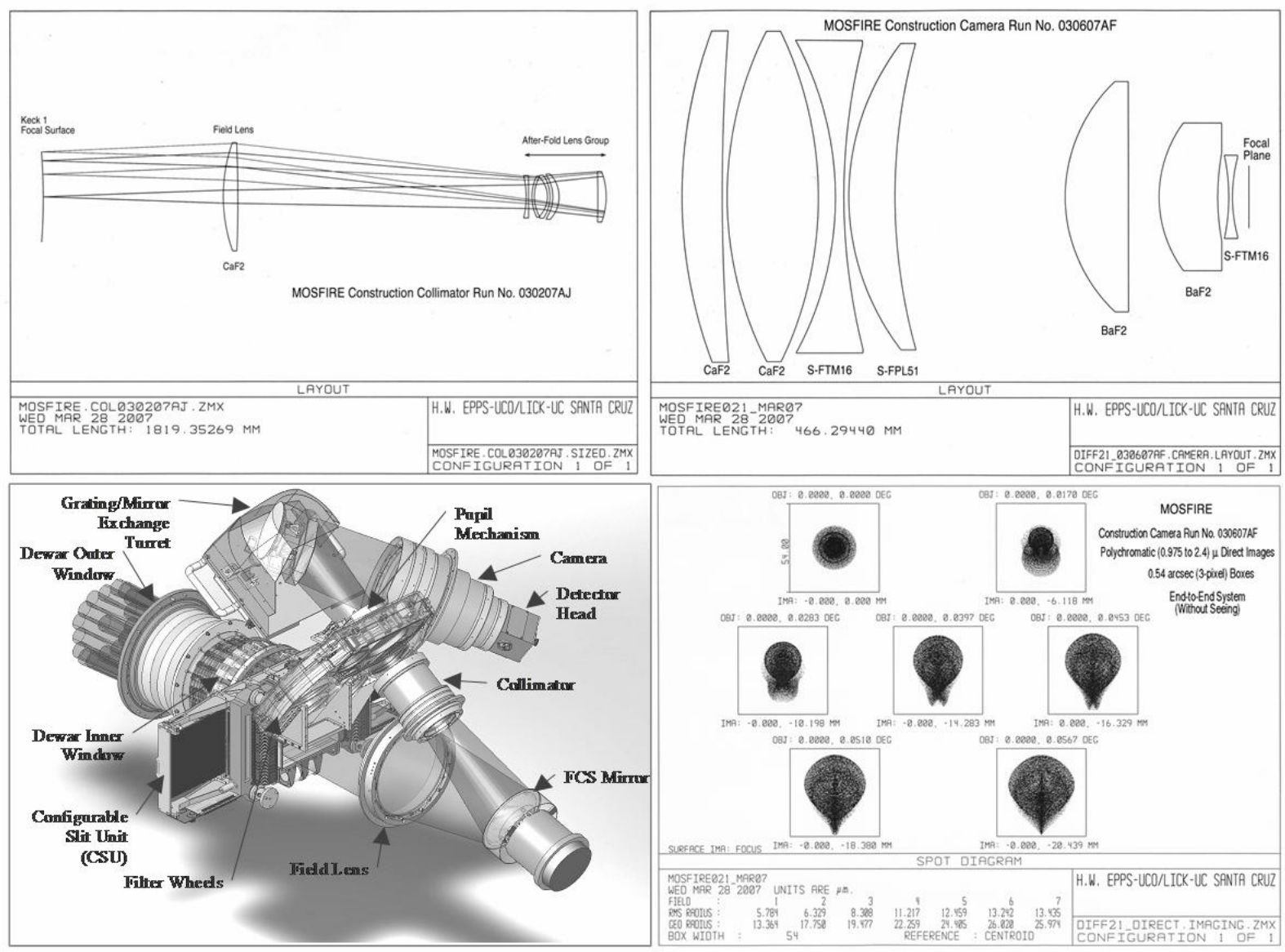

Fig. 2. MOSFIRE optical layout. Upper panels: Collimator and Camera sections. Lower left panel: physical layout. A double window precedes the CSU. The plane mirror in the folded Collimator is tip/tilt controlled to compensate flexure. Lower right panel: end-to-end polychromatic spot diagrams; 3 pixel box.

The 6-element collimator contains a large CaF2 field lens separated from a second lens group. A folding flat is placed between these groups. Curvature of the Keck focal plane ( $\sim 2.124 \mathrm{~m}$ radius) is taken into account in the CSU design. The pupil image is stationary over all field angles to $\sim 2.5 \%$ in the K-band and to $\sim 3.6 \%$ in the H-band where a Lyot stop is used. Filters are titled 6.0 degrees with respect to the optical axis to avoid ghost images. 
The large $(327.5 \mathrm{~mm})$ distance from the Lyot stop to the grating, combined with the $(300.0 \mathrm{~mm})$ distance from the grating to the camera causes convoluted, anamorphic, wavelength and field-angle dependent compound-pupil presentations, which complicate the design. The resulting camera requires a $250.0 \mathrm{~mm}$ focal length and a $270.0 \mathrm{~mm}$ entrance aperture such that it operates at $\mathrm{f} / 0.93$ (under-filled), which is unprecedented for a cryogenic $0.975 \mu \mathrm{m}$ to 2.40 $\mu \mathrm{m}$ infrared camera of these dimensions. Its field radius is 6.0 degrees to cover the corners of the H2-RG array. A 7element all-spherical camera design has been produced (by H. Epps) that satisfies all the goals for MOSFIRE, including the need to provide for direct imaging in the $\mathrm{K}, \mathrm{H}, \mathrm{J}$, and $\mathrm{Y}$ passbands as well as spectral coverage in those passbands for any object located within a rectangle at the telescope focal surface whose dimensions are $\pm 3.06^{\prime}$ along the slits and $\pm 1.50^{\prime}$ perpendicular to the slits.

The end-to-end direct-imaging performance of the telescope, collimator and camera combination is excellent, as seen in Figure 2 (bottom right panel). These polychromatic spot diagrams show a "wide-open" passband, which includes 20 wavelengths from $0.975 \mu \mathrm{m}$ to $2.40 \mu \mathrm{m}$ plotted simultaneously without refocus, for field angles that are $0.0,30 \%, 50 \%$, $70 \%, 80 \%, 90 \%$ and $100 \%$ of the full $6.8^{\prime}$ field of view that is available for direct imaging. The area-weighted average rms image diameters are only $0.23 " \pm 0.06$ " and all of the light falls within 3-pixel (0.54") boxes. Imaging will be even better in the $\mathrm{K}, \mathrm{H}, \mathrm{J}$, and Y passbands, which can be focused independently with the built-in focus mechanism.

The nominal grating angle of 42.614 degrees nicely centers spectra for slits at the center of the MOSFIRE field of view in the $\mathrm{H}$ and $\mathrm{K}$ bands, but the $\mathrm{Y}$ and $\mathrm{J}$ bands end up slightly "short" at the same angle. This fact led us to incorporate a second grating angle of 41.524 degrees that will be the default for observations in the $\mathrm{Y}$ and $\mathrm{J}$ bands. Using a simple mechanism that places a shim in front of the normal stop for the grating mechanism, the second angle is achieved without compromising grating repeatability. The specified grating blaze angle of 21.93 degrees attempts to optimize the location of the blaze peak with respect to the useful bandpass for each order. Finally, the flat mirror used for the imaging mode and mounted back-to-back with the grating has over-coated silver on the same substrate material used for the grating, OHARA Clearceram-Z.

\section{MECHANICAL DESIGN}

A sectional side view of MOSFIRE is shown in Figure 3. MOSFIRE is designed for mounting at the Cassegrain position of the Keck I telescope. This focal station imposes strict space envelope limitations as well as requirements to cope with varying gravity vectors during operation. The instrument design must meet specific weight and balance requirements and provide definition points compatible with the existing points used for the Low Resolution Imaging Spectrometer (LRIS) instrument on Keck I.

As mentioned, the entrance window to the vacuum enclosure (dewar) is a double window, with a thicker outer window providing the vacuum seal and a second inner window to limit thermal radiation reaching the CSU masking bars. A snout tube extension of the dewar aids in thermal isolation of the instrument by increasing the separation between the windows and the CSU, thus reducing the viewing angles. Heater elements are located between the windows within the snout tube. This heater is constructed of coated aluminum rings with unique profiles to efficiently heat the outer window while minimizing the heat directed to the inner window and CSU. Surfaces facing the outer window are coated with black paint and the surfaces facing the interior of the instrument are plated with gold. The heater/baffle assembly consists of strip heaters attached to the outer wall of this unit and covered with MLI. Attachment of the heater/baffle assembly to the snout is insulated to minimize heat transfer to the dewar walls, which therefore minimizes heater power requirements as heat is restricted to radiating to the outer window and snout interior rather than also trying to heat the dewar walls.

The dewar has tori-spherical vacuum caps at both ends. Both end caps are shaped for vacuum loading efficiency. Each end cap is removable for servicing, and the intent of the design is to make all of the mechanisms accessible for servicing after removal of either the front or the rear end cap. This dewar shape also helps to minimize weight in order to fit within the weight budget for this focal station. Like the front end cap, the rear end cap also has a cylindrical protrusion that in this case houses the flexure compensation system (FCS). The cable wrap fills the space between the rear protrusion and the surrounding rotator module. 


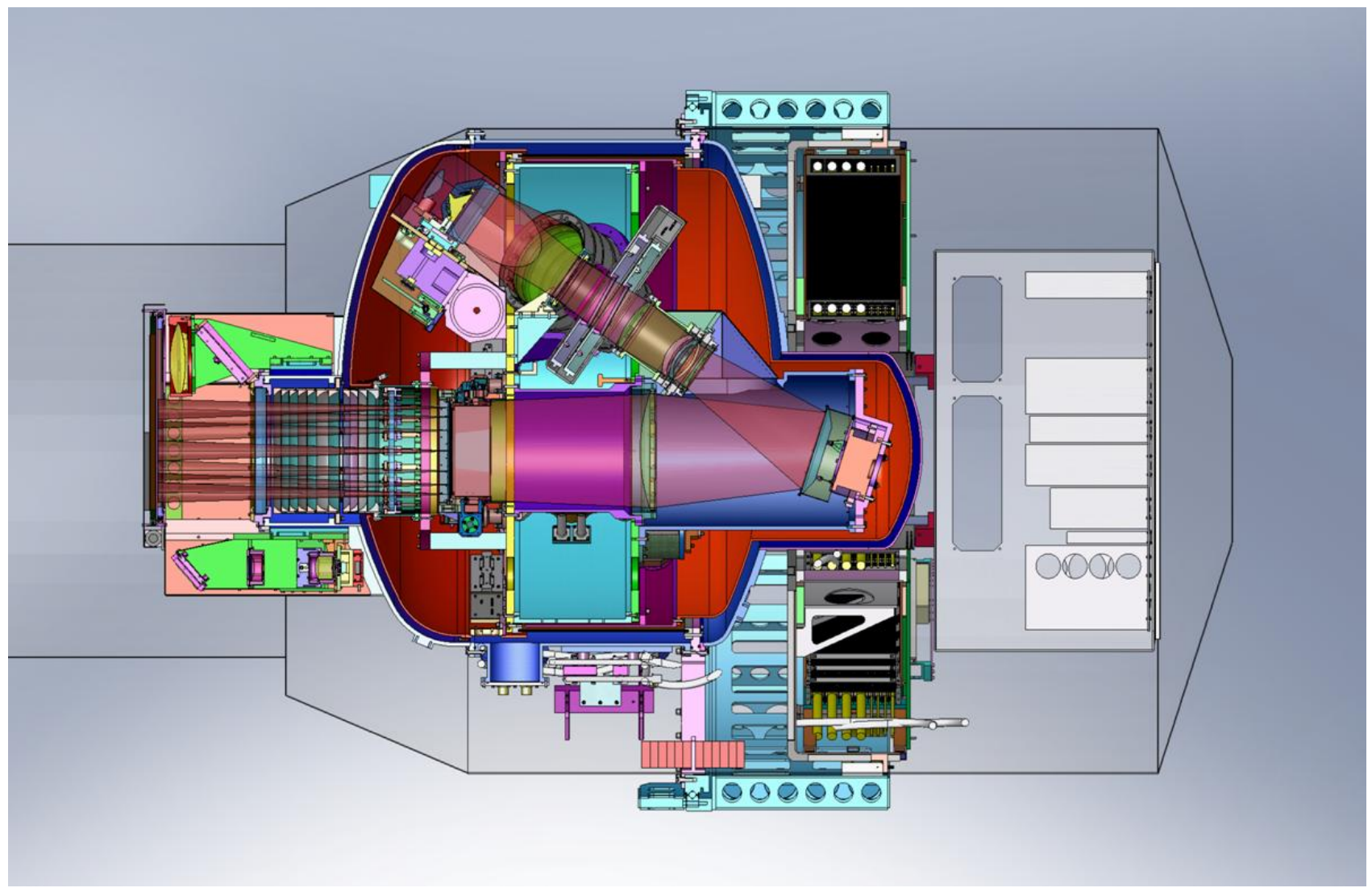

Fig. 3. A section view of MOSFIRE. The thin black outline indicates the Keck I Cassegrain envelope limits. The dewar is mounted forward of the rotator bearing and the Cable Wrap and electronics cabinets are mounted behind. The cylindrical dewar is about $5 \mathrm{ft}(1.5 \mathrm{~m})$ in diameter.

A CCD guide camera (2.8 $\times 2.8^{\prime}$ field of view) and arc-lamp calibration system is mounted on the front snout of the dewar. Flexure between the Guider and CSU, evaluated separately, was found to be negligible relative to the flexure of the vacuum chamber itself. Flexure of the CSU bars is negligible in the $\mathrm{x}$ and $\mathrm{z}$ directions, but the masking bars have been estimated to flex up to $\pm 200 \mu \mathrm{m}$ with gravity in y when a bar is fully extended; hence the large overlap of $700 \mu \mathrm{m}$ between bars to provide good margin at the expense of a shorter effective slit (5.1 $\mathrm{mm}$ compared to the $5.8 \mathrm{~mm}$ pitch). The design goals are equivalent to 0.1 " of relative motion, while the requirement is equivalent to 0.3 " of relative motion. In all cases but one, the relative flexure is predicted to be less than half of the goal. For the relative y displacement, the estimate is approximately twice the goal, but still lower than the requirement, with margin.

Lens mounts must hold the lenses in place to the required mounting tolerances while allowing for differential expansion and contraction during cool down cycles. For large optics like those used in MOSFIRE, typical approaches based on compliant cells can easily result in excessive stress on the optics. A design based on tangential "flexures" has been refined, prototyped and tested during the detailed design phase. This mount has been shown to provide negligibly small radial and axial motions of the lens element, with negligible hysteresis, as the mount is rotated and tilted. Lenses are separated into two groups, the Collimator group and the Camera group. The Camera group has an interface to the Detector Head and the Collimator is subdivided into the Field Lens and the 5-element Collimator Barrel. The alignment and interface methodology for these two lens units is the same. Cells are assembled together with a bolt pattern arrangement. Individual cells have adjustable spacer shims installed at each cell-to-cell interface and the spacer thickness can be altered at the last minute to account for as-built lens variations. In addition, these spacer shims have their internal diameters sized to serve as optical baffles within each barrel (Figure 4). 
MOSFIRE has a double Filter Wheel. Each wheel is supported by and revolves around a central hub using a 440C stainless steel, 4 point contact ball bearing from Kaydon Corporation. Using a 4-point-contact bearing allows us to support both the radial and axial loads using a single bearing per wheel. The central axle is made from stainless steel (416). The axle's coefficient of thermal expansion (CTE) is similar enough to the ball bearing's CTE that no additional compensation for differential expansion is needed for the interface between the bearing's inner diameter and the axle's outer diameter. The bearings are installed in a central well in the individual wheels. Since differential expansion between stainless steel and aluminum is an issue here, the clearance between the bearings and the wheels is slightly oversized for lab ambient temperature and optimized for operational temperature. Thin retaining rings constrain the bearings axially but act as springs during cool down, again to compensate for differential CTE's.
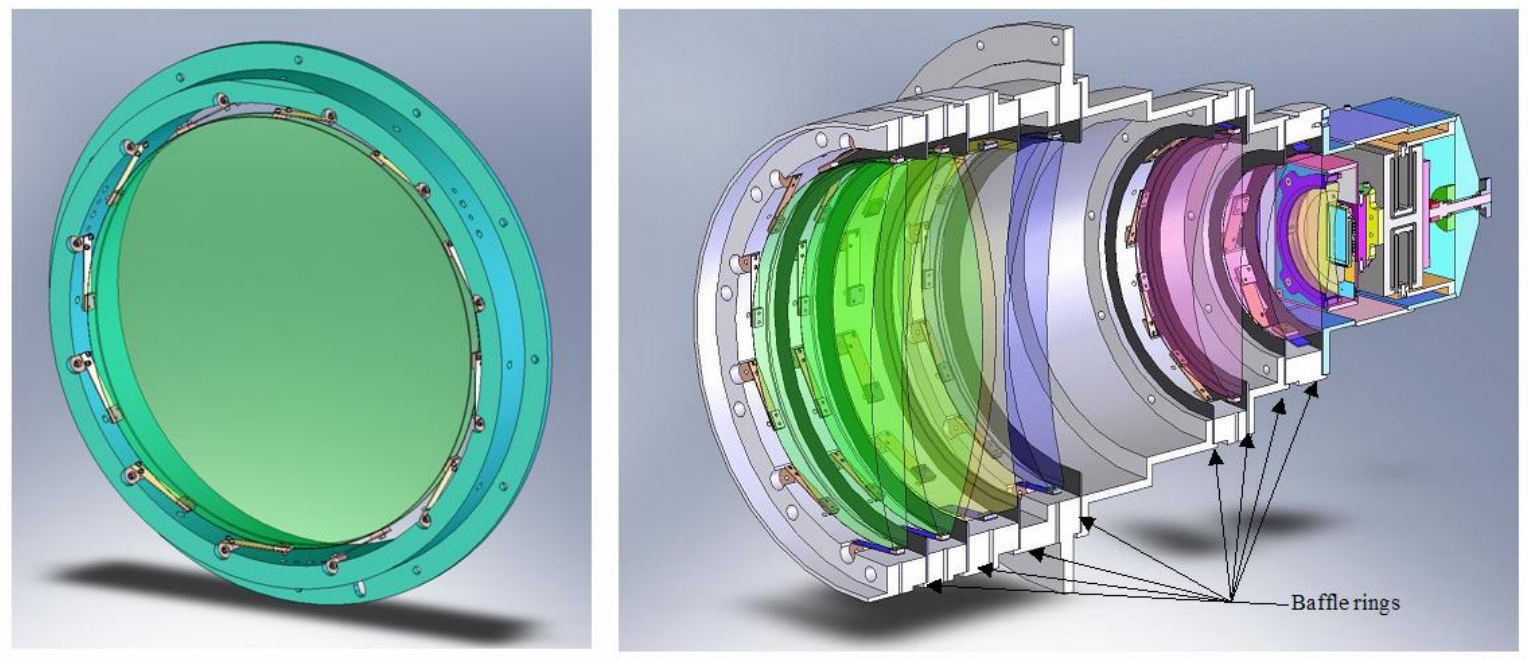

Fig. 4. Left: The "flexures" method of lens mounting. Right: Section view showing the MOSFIRE Camera Barrel assembly and interface to the detector.

The CSU is described in detail by Spanoudakis et al. elsewhere in these Proceedings ${ }^{4}$. Briefly, a slit is formed at a designated position within the FOV by positioning two opposing bars so as to create a rectangular opening. The end of each bar carries a slit jaw whose height (z-axis) is set to approximate the curvature of the Keck Telescope focal plane. A set of 46 bar pairs will be used to form the MOSFIRE focal plane mask. The sides of the bars are convoluted and there is a large, non-contact overlap $(0.7 \mathrm{~mm})$ so that light is prevented from passing between adjacent bars. Bars that form one side of the slit can be moved independently of those that form the other side, so that any width of slit can be formed, up to a completely open imaging field $(267 \times 267 \mathrm{~mm})$ when the bars are fully retracted.

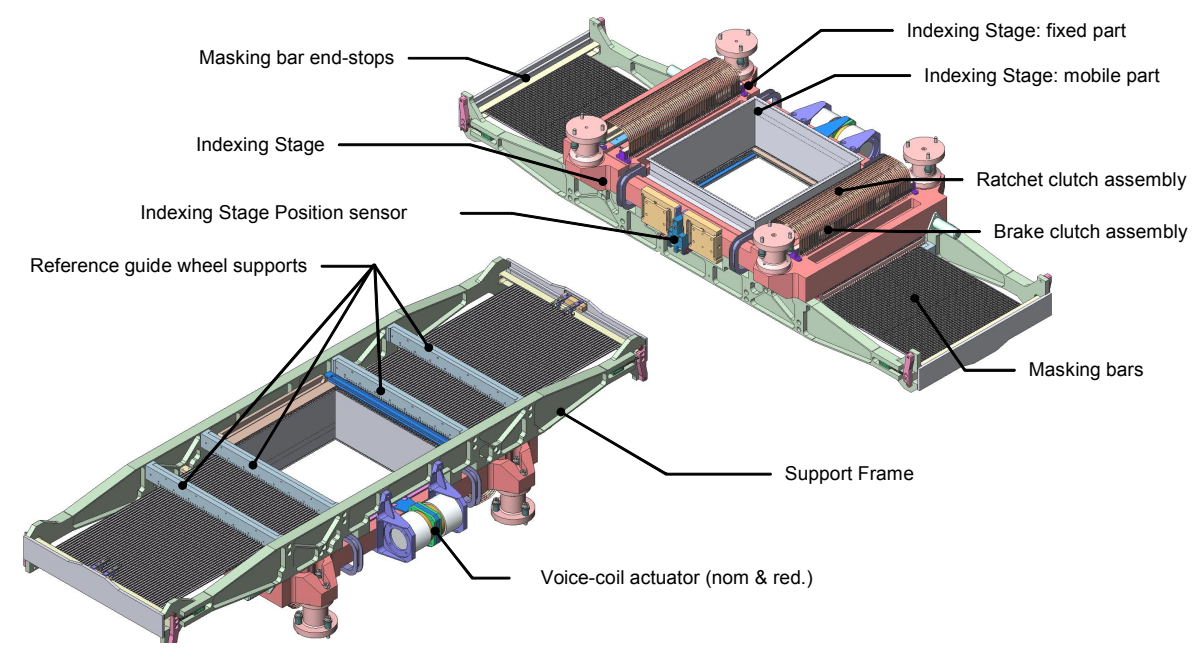

Fig. 5. Major CSU Sub-assemblies: the Indexing Stage (top) and Support Frame (bottom) 
The CSU consists of two major subassemblies, the indexing stage and the support frame. Figure 5 shows the full scale design of these two subassemblies in detail. It is the indexing stage that provides the mechanism for positioning the bars. Masking bars are displaced across the FOV using incremental steps (inchworm principle) where a number of oscillating steps are required to displace the masking bars. There are two modes of moving the bars, a coarse movement that can move many (all) bars simultaneously, and a fine motion that adjusts each bar individually to the correct inter-tooth position. A voice-coil actuator on an indexing stage provides the motion to move the bars from opposite sides simultaneously. A common position sensor (LVDT) keeps track of all relative movement of bars with respect to the fixed reference frame.

Removal of the front cover of the MOSFIRE vacuum enclosure provides immediate access to four sub-assemblies. These are the CSU, the grating/mirror turret, the window heater assembly, and the inner window module (Figure 6). The left and right main refrigerator head thermal straps can also be reached from the front through access holes on Bulkhead A, but access from the rear is better due to larger access holes in the rear (B) bulkhead. A sixth sub-assembly, the pupil mechanism, can be reached once the grating/mirror turret has been removed.
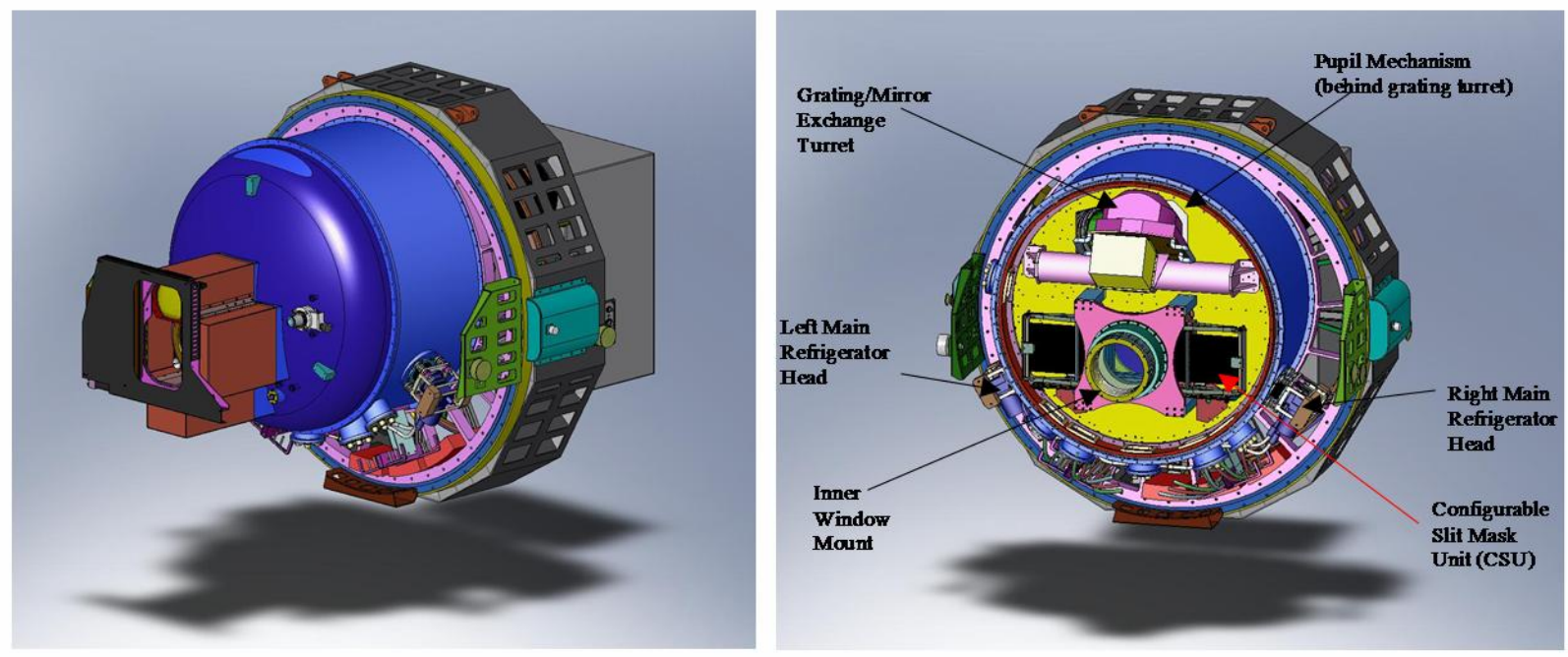

Fig. 6. MOSFIRE with the front cover and shields removed. Accessible sub-assemblies are indicated.

\section{CONTROL SYSTEMS AND SOFTWARE}

MOSFIRE's electronics design is based on the heritage of recent WMKO instruments, in particular OSIRIS, NIRSPEC and NIRC2. Most of the electronics consist of commercial off the shelf (COTS) equipment with the required interconnections. There is a limited amount of custom equipment, including the CSU control system, the interface board for the science detector's application specific integrated circuit (ASIC) and a few printed circuit boards used to simplify various high pin count interconnections. MOSFIRE electronics consists of three major component groups, the instrument electronics located in the electronics cabinet mounted on the instrument; the MOSFIRE CCR compressor and controls located in the Keck I telescope mechanical equipment room; and the MOSFIRE computer rack containing the instrument host computer and data storage disks located in the Keck I Telescope computer room.

The $2 \mathrm{~K} \times 2 \mathrm{~K}$ H2-RG ASIC, called "SIDECAR", provides clocks and bias voltages to the detector and digitizes the detector outputs. The ASIC chip is packaged separately on a small board that is located inside the dewar next to the detector head. Just outside the dewar wall is another board, the Jade2, which provides the interface between the ASIC and USB 2.0. A computer running Windows XP is required with a USB interface. This computer, which is equipped with a flash drive and rack-mounted in one of the MOSFIRE electronics cabinets, will function as the detector control computer. It will be located on a private network within the instrument and will not be accessible via network communications except through the MOSFIRE host computer. 
MOSFIRE will benefit from a polished and robust client-server architecture, which facilitates distributed processing and remote observing. This framework began with NIRSPEC and NIRC2, and was most recently refined in the implementation of the OSIRIS instrument software. The server architecture offers many features that have become standard practice for infrared instruments at WMKO. Each instrument server is compliant with the Keck Task Library (KTL), which uses remote-procedure calls (RPCs) in a WMKO standard framework for network communications. Standard WMKO keywords are used as the main applications programming interface (API), and simple clients, such as "show" and "modify" facilitate easy access and scripting. By using existing KTL interfaces to other popular languages such as Java, IDL and Tcl, a diverse range of user interfaces can be implemented.

At the time of writing, MOSFIRE is in the middle of the construction phase and many of the sub-systems described here are nearing completion and will soon be ready for integration and testing.

\section{ACKNOWLEDGMENTS}

It is a pleasure to thank all the members of the MOSFIRE team and our sub-contractors, as well as the members of our review boards for their many contributions and support. This material is based on work funded by the generous financial support of Gordon and Betty Moore and supported by AURA through the National Science Foundation under AURA Cooperative Agreement AST 0132798, as amended. This work was performed for the W. M. Keck Observatory, which is operated as a scientific partnership among the California Institute of Technology, the University of California and the National Aeronautics and Space Administration. The Observatory was made possible by the generous financial support of the W. M. Keck Foundation.

\section{REFERENCES}

[1] McLean, I. S. and Adkins, S. M., “Instrumentation Development at the W. M. Keck Observatory," Proc. SPIE 6269, 1-12 (2006).

[2] Kibrick, R. I.; Miller, J. S., Nelson, J. E.; Radovan, M. V.; Sheinis, A. I.; and Sutin, B. M., "Active flexure compensation software for the Echellette Spectrograph and Imager on Keck-II," Proc. SPIE 4009, 262-273 (2000).

[3] Kibrick, R. I.; Faber, S. M.; Phillips, A. C.; McVeigh, M.; Cowley, D. J.; Radovan, M. V.; Gilmore, D. K.; Wright, C. A.; Tucker, D.; Clarke, D.; and Allen, S. L., "A comparison of open versus closed loop flexure compensations for two Keck optical imaging spectrographs: ESI and DEIMOS,” Proc. SPIE 4841, 1385-1398 (2003).

[4] Spanoudakis, P.; Giriens, L.; Henein, S.; Lisowski, L.; O’Hare, A.; Onillon, E.; Schwab, P.; and Theurillat, P., "Configurable slit-mask unit of the Multi-Object Spectrometer for Infra-Red Exploration for the Keck telescope: Integration and Tests," Proc. SPIE 7018 (2008). 